19 Revue d'histoire du XIXe siècle

Société d'histoire de la révolution de 1848 et des

révolutions du XIXe siècle

32 | 2006

Varia

\title{
Le mot du président
}

Jean-Claude Caron

\section{OpenEdition}

\section{Journals}

Édition électronique

URL : http://journals.openedition.org/rh19/1087

DOI : $10.4000 /$ rh 19.1087

ISSN : $1777-5329$

Éditeur

La Société de 1848

Édition imprimée

Date de publication : 1 juin 2006

ISSN : 1265-1354

Référence électronique

Jean-Claude Caron, « Le mot du président », Revue d'histoire du XIXe siècle [En ligne], 32 | 2006, mis en ligne le 12 juillet 2006, consulté le 22 septembre 2020. URL : http://journals.openedition.org/ rh19/1087 ; DOl : https://doi.org/10.4000/rh19.1087 


\section{JEAN-CLAUDE CARON Le mot du président}

Ces quelques lignes n'ont pas pour objet de présenter ce numéro : ses responsables, Judith Lyon-Caen et Manuel Charpy, s'en acquittent de belle manière, de même qu'ils ont su mener à terme ce Varia dont le contenu, nous l'espérons, satisfera nos lecteurs. C'est aussi une manière de souligner que la forme même des numéros Varia implique une diversité d'écriture et de thématique qui reflète l'état actuel de la recherche historique. Nous sommes intéressés à recevoir des articles en témoignant : que leurs auteurs n'hésitent donc pas à nous les adresser. Plus généralement, ce numéro 32 de la Revue d'histoire $d u$ XIXe siècle, qui est le premier de l'année 2006, me donne l'occasion de présenter un bilan de l'année écoulée. Je le fais d'autant plus volontiers que nos adhérents ne peuvent pas nécessairement assister à l'Assemblée générale de notre Société. Je souhaite donc les informer de nos activités et des décisions qui ont été prises. Et j'ajoute que, si j'assume seul la teneur des propos qui suivent, c'est bien d'une activité collective dont il va être question : celle des membres qui, bénévolement, consacrent de leur temps, au sein du Bureau, du Conseil d'administration, ou du Comité de rédaction de la $R H 19$, à la gestion de cette Société et de sa revue. Les rubriques qui suivent me donneront l'occasion de les citer, mais je voudrais dès maintenant rappeler que le rôle de la secrétaire générale, Sylvie Aprile, est essentiel dans le bon fonctionnement de cette Société.

Devant initialement se tenir le samedi 18 mars 2006, l'Assemblée générale de notre Société s'est finalement tenue le 13 mai 2006, après la réouverture de la Sorbonne. Le Rapport moral que j'ai présenté a été l'occasion de rappeler quelques réalisations de l'année 2005 :

- L'organisation d'une journée d'étude consacrée à la question d'histoire contemporaine proposée aux étudiants préparant les concours du Capes et de l'agrégation ("Les campagnes dans les évolutions sociales et politiques en Europe, des années 1830 à la fin des années 1920 : étude comparée de la France, de l'Allemagne, de l'Espagne et de l'Italie») nous a permis de réunir un public nombreux dans l'amphithéâtre Richelieu de la Sorbonne le 
15 octobre 2005; un volume, reprenant les communications présentées ce jour, ainsi que d'autres, a été publié aux Presses universitaires de Rennes, grâce à l'intermédiaire de Frédéric Chauvaud.

- Deux numéros de la Revue d'histoire du XIXe siècle ont été publiés : le $\mathrm{n}^{\circ} 30$, dirigé par Odile Roynette, propose une réflexion collective innovante, "Pour une histoire culturelle de la guerre au XIXe siècle»; le second proclame que "La «Société de 48» a cent ans» et reprend les communications présentées à l'occasion de la journée commémorant le centenaire de notre Société en 2004. Je rappelle que ce dernier numéro comporte également les Tables décennales de la Revue pour les années 1995-2004 : comme pour les précédentes tables, nous les devons à Jean-Claude Farcy, qui a effectué un remarquable travail. Ces deux numéros ont été pris en charge par Louis Hincker, qui a souhaité passer la main : je souhaite le saluer et le remercier pour la qualité du travail effectué en tant que secrétaire de rédaction de la Revue d'histoire du XIXe siècle. Ce numéro 32 est le premier qui parait sous la seule responsabilité de Fabrice Bensimon, qui avait déjà travaillé en tandem avec Louis Hincker pour le précédent numéro. Je signale également que Judith Lyon-Caen est désormais responsable de la rubrique «Comptes rendus».

- Le changement d'imprimeur et le recours au service d'un maquettiste ont modifié nos façons de faire : globalement, le relais s'est correctement passé. La réussite de cette transition tient aussi au fait que la fabrication des deux numéros annuels de la RH19 relève d'un travail collectif. Nous souhaitons par ailleurs paraitre de manière plus régulière et limiter le volume de chaque numéro : il en va de notre crédibilité et de notre pérennité. Car, il convient de le rappeler, notre situation financière demeure fragile et seule la subvention versée par le CNL nous permet de financer la RH19- les cotisations de nos adhérents n'y suffisant pas. Le deuxième numéro de l'année 2006, piloté par Louis Hincker et Jacques Rougerie, est consacré à «Relations sociales et espace public». Il pourrait d'autant mieux donner lieu à un prolongement sous forme d'une journée d'étude qu'un des deux numéros de l'année 2007 sera consacré à la bourgeoisie (titre provisoire de ce numéro coordonné par Sylvie Aprile, Manuel Charpy et Judith Lyon-Caen). L'idée d'une réflexion sur la catégorisation en histoire sociale a été lancée, en association avec Michèle Riot-Sarcey.

- Grâce à Carole Christen-Lécuyer, le site Internet de notre revue (rh19. revues.org) a connu une refonte totale au plan de sa forme et des ajouts conséquents au plan de son contenu. Nous souhaitons amplifier cet enrichissement : ainsi, par exemple, publier des positions de thèse, des documents, etc. Par ailleurs, si, de manière exceptionnelle, le numéro consacré au centenaire de la Société de 1848 a été intégralement mis en ligne, nous continuons à mettre partiellement en ligne les autres numéros. Mais nous sommes arrivés à une phase nouvelle de notre réflexion sur le passage à une éventuelle mise 
en ligne payante de nos numéros les plus récents. Des modifications des statuts de notre hébergeur (revues.org) sont à prévoir, nous permettant, si nous le souhaitions, de réfléchir à cette possibilité. Plus généralement, il semble que les débats sur la relation entre la version papier et la version électronique des revues savantes arrivent à un stade décisif, où la question des financements publics, sous forme de subvention, devient centrale dans la réflexion. Nous suivons tout cela de près, étant concernés au premier plan.

- Le colloque "Violence et conciliation en Europe au XIXe siècle. Une histoire de la résolution des conflits socio-politiques" aura lieu du 25 au 27 janvier 2007 : le premier jour à l'université de Paris XII-Créteil, qui nous accueillera grâce à l'amitié de Florence Bourillon et d'Emmanuel Fureix; les deux jours suivants à la Maison de la Recherche de Paris IV, dont l'accueil a été rendu possible par l'intervention de Jean-Noël Luc. Une trentaine de propositions de communication ont été retenues par le Comité d'organisation, composé de Sylvie Aprile, Frédéric Chauvaud, Laurent Colantonio, Emmanuel Fureix, Jacqueline Lalouette, Jean-Noël Luc et moi-même. La prochaine étape est la mise au point d'un programme provisoire. Quant au financement, outre les fonds propres de la Société, il sera assuré par des aides d'ores et déjà acquises : celles du Centre de recherches en histoire du XIXe siècle de Paris I et Paris IV (Jean-Noël Luc), de l'Université de Poitiers (Frédéric Chauvaud), du Centre d'histoire Espaces et Cultures (Université Blaise-Pascal, ClermontFerrand, Jean-Claude Caron). D’autres pistes sont explorées.

- Le séminaire organisé par la Société de 1848 ne sera pas renouvelé l'année prochaine. Les sept séances de l'année 2005-2006 n'ont pas suscité suffisamment d'intérêt de la part de nos adhérents pour que nous poursuivions, en l'état, cette activité. Cet échec a des raisons multiples, allant d'une thématique peut-être mal définie ou trop identique à celles déjà couvertes par d'autres séminaires; à la concurrence très rude des activités de ce genre à Paris, spécialement le mercredi ; à la difficulté de constituer un noyau suffisamment nombreux de membres de notre Société autour d'un séminaire mensuel. Cela posé, toutes les suggestions peuvent être étudiées pour la reprise de ce dernier dans deux ans, à partir d'un projet qui reste à élaborer.

- Présenté par Christophe Voilliot, le rapport financier constitue un temps fort de chaque assemblée générale. Parce qu'il confirme la fragilité de nos finances, l'existence d'un déficit chronique et conditionne donc la suite de nos activités; mais aussi parce qu'il nous contraint à imaginer des solutions nous permettant de faire des économies. L'une sera réalisée prochainement : grâce à Judith Lyon-Caen, nous disposerons d'un lieu de stockage nous permettant d'économiser les frais de location d' "Une pièce en plus", soit environ 1000 euros par an. Nous avons d'autre part arrêté la décision de limiter les numéros de la RH19 à 250 pages par numéro, ce qui correspond à un standard assez largement partagé par les revues comparables à la nôtre. Enfin, si besoin était, nous pourrions envisager, certaines années, un numéro 
double en lieu et place de deux numéros : en conservant la même pagination, soit autour de 500 pages annuelles, l'économie n'est pas négligeable en termes de fabrication et de frais postaux. La solution à ces insuffisances de revenus propres à notre Société est simple et formulée depuis longtemps : augmenter le nombre de nos adhérents. Aussi lancé-je de nouveau un appel pour que chacun fasse connaître notre existence, en particulier auprès des institutions qui pourraient souscrire un abonnement (archives départementales, bibliothèques universitaires) ou auprès des étudiants à qui est réservé un tarif spécifique.

- Ceci m'amène à vous informer que, à la suite de la décision adoptée en Conseil d'administration le 25 novembre 2005 et votée à l'unanimité par l'Assemblée générale du 13 mai 2006, les tarifs d'adhésion sont modifiés ainsi que suit, à partir de l'année 2007 : 38 euros pour les individuels (42 euros hors Union européenne); 42 euros pour les collectivités (48 euros hors Union individuelle); 24 euros pour les étudiants (sur présentation d'un justificatif). Le niveau de ces tarifs se situe dans la moyenne de ceux des autres sociétés savantes.

Au terme de ce "Mot du président", je voudrais rappeler que seule la fidélité de nos adhérents nous permet d'exister. Je les remercie donc très sincèrement de nous manifester encore et toujours cette fidélité et leur demande de ne pas hésiter à faire connaître notre existence et nos activités. C'est ainsi et seulement ainsi que nous assurerons notre indépendance et notre pérennité.

Jean-Claude Caron, président de la Société d'histoire de la révolution de 1848 et des révolutions du XIX siècle 